\title{
Prevalence and Factors Associated with Enuresis among Children in Adama City, Oromia Regional State, Ethiopia
}

\author{
Nuru Hassen Ibrahim*, Daniel Tolessa and Ephrem Mannekhulihe, MSc \\ School of Public Health Department, Adama Hospital Medical College, Adama, Ethiopia

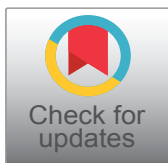 \\ *Corresponding author: Nuru Hassen Ibrahim, Principal Investigator, Assistant Professor, School of Public Health \\ Department, Adama Hospital Medical College, Adama, Ethiopia
}

\begin{abstract}
Enuresis is voluntary or involuntary voiding of urine, occurring at least twice a week for 3 consecutive months in a child at least five-years-old. It can occur either during the day or at night time. There are two types of enuresis called primary and secondary enuresis. The word enuresis is derived from a Greek word enourein that means "to void urine."
\end{abstract}

Objective: To assess the prevalence and factors associated with enuresis among 6-16 years-old age children in Adama city.

Method: Community based cross sectional study was carried out in the Adama city in all 18 kebele. An interview questionnaire was completed voluntarily by the children of 6-15 year-old and with their parents during data collection. The duration of study was from April 2018 to June 2018.

Results: A total of 866 children were included in the study. The prevalence rate of enuresis in Adama city among children age $6-15$ was $26.6 \%$. Similarly in this study prevalence of enuresis among 7 years children is $30 \%, 10$ years children is $25 \%$ and $20 \%$ among 14 years children. The mean age of enuretic age children is 9.95. Prevalence of enuresis is more common among males than female. According to this conducted study in Adama city enuresis prevalence among age 6- 16 years is $76.09 \%$ [175] for boys and 23.91 [55] among girl children. it was found that the mothers of $55.13 \%$ [113] had the problem of enuresis while $16.59 \%$ [34] father, $17.07 \%$ [35] siblings , $10.24 \%$ [21] cousin and $0.97 \%$ [2] had enuretic relatives. In this study primary enuresis encounter $91.3 \%$ [210] and $8.7 \%$ [20] for secondary enuresis from the total of 230 enuretic children in Adama city. Only parents of $53.04 \%$ [122] children with enuresis had consulted a medical doctor about it. $96.52 \%$ [222] enuretic children do have more than one sibling. The $69.13 \%$ [159] enuretic children's are second and above born in the family.
Conclusion: The study suggests that enuresis is a common problem among 6-16 years-old children in Adama city and its prevalence is higher than study conducted result in the literature. Positive family history of enuresis, number of siblings in household, birth order in the family, lower age, punishment of children by their parents, underlying medical and psychiatric illness, were identified as the associated risk factors for enuresis.

\section{Keywords}

Children, Enuresis, Factors, Prevalence

\section{Background of the Study}

The word enuresis is derived from a Greek word, enourein that means "to void urine" [1]. The International Children's Continence Society restricts the term to wetting that occurs at night. Enuresis can be divided into primary enuresis and secondary enuresis. A child who was a continent for at least 6 months before the onset of the bedwetting is considered to have secondary enuresis. The pathogenesis of primary enuresis is similar to that of secondary enuresis. In primary enuresis, psychological problems are almost always the result of the condition in only rarely the cause. In secondary enuresis, however, psychological problems are a possible cause, albeit not a common one. The Co-morbidities of behavioral problems are two to four times higher in children with enuresis [2].

Enuresis is repeated voiding of urine into bed or cloths whether involuntary or intentional, The behavior is clinically significant as manifested by either a frequency of twice a week for at least 3 consecutive months or the

Citation: Ibrahim NH, Tolessa D, Mannekhulihe E (2021) Prevalence and Factors Associated with Enuresis among Children in Adama City, Oromia Regional State, Ethiopia. Int J Physiatry 7:021. doi. org/10.23937/2572-4215.1510021

Accepted: November 20, 2020: Published: November 22, 2020

Copyright: (c) $2020 \mathrm{lbrahim} \mathrm{NH}$, et al. This is an open-access article distributed under the terms of the Creative Commons Attribution License, which permits unrestricted use, distribution, and reproduction in any medium, provided the original author and source are credited. 
presence of clinically significant distress or impairment in social, academic, occupational, or the behavior is not due exclusively to the direct physiological effect of a substance for example, a diuretic or a general medical condition like diabetes, spinal bifida, or a seizure disorder [3].

Worldwide, the prevalence of primary enuresis seems to be approximately the same, though no standardized evaluation of the prevalence of bedwetting has been made on a global basis [4]. However, diagnostic and statistical manual of American psychiatric disorder classification states the prevalence of enuresis as 5\%$10 \%$ among 5-year-olds, 3-5\% among 10-year-olds, and around $1 \%$ among individuals 15 years or older [5].

In Ethiopia, conflicting estimates of the prevalence of enuresis in children have been reported by different studies: $5 \%$ in a small town [6], $0.8 \%$ in a predominantly rural sample [6] and $9 \%$ in a group of urban child laborers and their controls [7]. None of these studies examined the association between enuresis and other demographic, social or psychopathologic factors. During 2002-2003, a cross-sectional survey of psychiatric disorders was carried out on children aged 6-15 years in Addis Ababa, Ethiopia, with diagnostic assessment according to the revised third edition of the Diagnostic Statistical Manual criteria. The results of that survey showed that enuresis had by far the highest prevalence of all conditions. While the weighted prevalence for any DSM-IIIR diagnosis was $16.9 \%$, the weighted prevalence of enuresis alone amounted to $12.3 \%$ [8].

When primary enuresis and secondary enuresis are reported, a secondary onset accounts for about 25\% of cases. The prevalence of secondary enuresis as a percentage of all cases increases with age. In a cohort of New Zealand children, $7.9 \%$ developed secondary enuresis by the age of 10 years [4]. Enuresis is more common in males. The reported prevalence of enuresis at the ages of 7 and 10 years is $9 \%$ and $7 \%$, respectively, in boys and $6 \%$ and $3 \%$, respectively, in girls. No racial predisposition has been documented [4].

Enuresis may be caused by many factors involved in the development of children's [9]. Enuresis Involuntary, or non-intentional, release of urine may result from: small bladder, persistent urinary tract infection, severs stress and developmental delays that interfere with toilet training, Voluntary, or intentional, enuresis may be associated with other mental disorders, including behavior disorders or emotional disorders such as anxiety [9]. Enuresis also appears to run in families, which suggest that a tendency for the disorder may be inherited, passed on from parent to child, particularly on the father's side. In addition, toilet training that was forced or started when the child was too young may be a factor in the development of the disorder, although there is little research to make conclusions about the role of toilet training and the development of enuresis.
Children with enuresis are often described as heavy sleepers who fail to awaken at the urinary urge to void or when their bladders are full [9].

\section{Objective of the Study}

\section{General objective}

$\checkmark$ To assess the prevalence and factors associated with enuresis among children in Adama city.

\section{Specific objectives}

$\checkmark$ To determine the prevalence of enuresis among children in Adama city.

$\checkmark$ To identify factors associated with enuresis among children in Adama city.

\section{Methods and Materials}

\section{Study design and period}

Community based cross sectional study designs were employed.

\section{Population}

Source population: The source population were all children living in Adama city.

Study population: The study population was children living in selected kebeles of Adama city during the study period.

\section{Eligibility criteria}

Inclusion criteria: Children aged 5-16 years was included in the current study.

Exclusion criteria: The study excluded children with acute illnesses as a result of medical illness, non-communicative children, and those who refused interviews. We also exclude the subjects who have been diagnosed as enuresis and being on treatment.

\section{Sample size determination}

The sample size was determined by using a formula for estimation of a single population proportion considering the following assumptions:

$Z=1.96$ : The corresponding standardized value of normal distribution at $95 \%$ confidence interval;

$P=0.09:$ The prevalence of enuresis in cities of Ethiopia has been reported to be $9 \%$ in big cities;

$d=0.02$ : The maximum level of tolerable error in estimating outcome of interest;

$\mathrm{n}=$ minimum required sample size

Accordingly the minimum required sample size determined as follow;

$$
n=\frac{\left(Z \frac{\alpha}{2}\right)^{2}(P)(1-p)}{d^{2}}=\frac{(1.96)^{2}(0.09)(0.91)}{(0.02)^{2}}=787
$$


Adding $10 \%$ non-response rate finally 866 subjects planned to be incorporated in the current study; $\mathrm{nf}=$ $787+79=866$ patients.

\section{Sampling procedure}

Adam city is divided in six sub-cities named as Abageda, Boku, Dabesoloke, Lugo, Bole, and Dembela. Regarding the prevalence of eneurosis all sub cities are assumed to be heterogeneous. Therefore all sub-cities were selected for the current study. Each sub-cities are further subdivided into three kebeles. One kebele from each sub-cities was randomly selected. Equal sample size was allocated for each kebele and according 45 children from each kebele were selected. The list of household in each selected kebeles was received from administrative office and household were selected using simple random sampling from the list. From children aged 5-15 years were available at household level one was randomly selected. Refusal to participate in the study and unavailability of eligible child were compensated by the next household.

\section{The variable}

The dependent Variable is the prevalence of enuresis and the independent Variables are sex, age, birth of new siblings, Educational status, Parental marital status, Parental Occupation, Family size, Corporal punishment, and Monthly income of the family.

\section{Operational definition}

- Enuresis - Involuntary or intentional, repeated voiding of urine into a child's clothes or bed; at developmental or chronological age of at least 5 years. The behavior must occur twice weekly for a period of at least 3 months or must cause distress and impairment in functioning to meet the diagnostic criteria. Enuresis is diagnosed only if the behavior is not caused by a medical condition.

- Prevalence - Is the proportion of a population who have a specific characteristic in a given time period.

- Factors - Elements contributing to a particular result or situation.

- Children - A young human being below the age of puberty or below the legal age of majority.

\section{Data collection tool}

The tool applied in this study was adopted from Jeffrey $E$, Lazarus, and $M, D$, board certified in pediatrics, child and adolescent clinical hypothesis and biofeedback. The questionnaire applied in the study consisted of two parts. The first part contained the demographic and socioeconomic data, including age, sex, parental marital status, parental kinship, parental occupation, family size, birth order, and other variables such as new baby's birth in the family, change of living place, sleep quality, child's corporal punishment by parents, academic failure, and corporal punishment at school. The second part consists of various kinds of active enuresis, nocturnal enuresis, diurnal enuresis, or both, primary and secondary enuresis.

\section{Data collection method}

An interview questionnaire was completed voluntarily by the children of $5-16$ year-old and with their parents during data collection. The study was conducted by Department of psychiatry in conjunction with social and public health department, Adama Hospital Medical College. The duration of study was from April 2018 to June 2018. The study was community based cross sectional study. It was carried out in the Adama city in all 18 kebele. Data was obtained according to a questionnaire. The data collectors' was trained to gather data accordingly for 866 children of 5-16 years included in the study.

\section{Data quality assurance}

To ensure data quality standard questionnaire was adapted from literatures of similar studies. Corrections and modifications were made. Data collection process was monitored. Training was given for data collectors and supervisors on data collection tools and procedures. Data was checked for completeness and consistency. Any incomplete information was checked and completed during supervision.

\section{Data processing and analysis}

The collected data was transferred in to Epi info version 7 then exported to SPSS version 20 for analysis. Before analysis data cleaning, categorizing and coding were done. Descriptive analysis was performed to explore the characteristics of study participants. Binary logistic regression analysis was used to identify the factors associated with Enuresis. Bi-variate logistic regression analysis was undertaken to select candidate independent variables associated with Enuresis. At this level P-Value less than 0.25 was used as cutoff point for selection of variables. Multivariate logistic regression analysis was used to estimate the adjusted effects of independent variables on Enuresis. The magnitude of association between independent and dependent variable was estimated using Odds Ratio (OR) with $95 \% \mathrm{Cl}$ and the significance of association were declared at $\mathrm{P}$-value less than 0.05 .

\section{Ethical consideration}

To conduct the study, the research proposal was examined and screened for scientific and ethical clearance by institutional review board for approving research in the college of medical science, Adama Hospital Medical College. Prior to administering the survey and collecting data, permission letter were given to, Adama city municipality and authorities of different levels, in Adama city where the study was 
Table 1: Showing characteristics of enuresis.

\begin{tabular}{|c|c|c|c|}
\hline No. & Variables & Frequency & Percentage \\
\hline \multirow[t]{4}{*}{1} & Enuresis type & & \\
\hline & Primary & 210 & 91.3 \\
\hline & Secondary & 20 & 8.7 \\
\hline & Total & 230 & 100 \\
\hline \multirow[t]{4}{*}{2} & Sought of Rx & & \\
\hline & Yes & 174 & 75.65 \\
\hline & No & 56 & 24.35 \\
\hline & Total & 230 & 100 \\
\hline \multirow[t]{5}{*}{3} & Treatment type & & \\
\hline & Modern & 156 & 89.7 \\
\hline & Traditional & 11 & 6.3 \\
\hline & Spiritual & 7 & 4.0 \\
\hline & Total & 174 & 100 \\
\hline \multirow[t]{3}{*}{4} & Punishment & & \\
\hline & Yes & 159 & 65.65 \\
\hline & No & 79 & 34.35 \\
\hline \multirow[t]{4}{*}{5} & $\begin{array}{l}\text { Prohibited café at } \\
\text { night time }\end{array}$ & & \\
\hline & Yes & 169 & 73.48 \\
\hline & No & 61 & 26.52 \\
\hline & Total & 230 & 100 \\
\hline \multirow[t]{4}{*}{6} & $\begin{array}{l}\text { Family history of } \\
\text { enuresis }\end{array}$ & & \\
\hline & Yes & 205 & 89.13 \\
\hline & No & 25 & 10.87 \\
\hline & Total & 230 & 100 \\
\hline \multirow[t]{7}{*}{7} & $\begin{array}{l}\text { Relationship of } \\
\text { enuretic Children } \\
\text { with others }\end{array}$ & & \\
\hline & Father & 34 & 16.59 \\
\hline & Mother & 113 & 55.13 \\
\hline & Siblings & 35 & 17.07 \\
\hline & Cousin & 21 & 10.24 \\
\hline & Others & 2 & 0.97 \\
\hline & Total & 205 & 100 \\
\hline \multirow[t]{3}{*}{8} & $\begin{array}{l}\text { Co morbidity to } \\
\text { Medical psychology }\end{array}$ & & \\
\hline & Yes & 192 & 83.48 \\
\hline & No & 38 & 16.52 \\
\hline \multirow[t]{5}{*}{9} & $\begin{array}{l}\text { Educational level } \\
\text { Enuresis }\end{array}$ & & \\
\hline & Low & $63 / 55 / 118$ & $27.39 / 13.6$ \\
\hline & Medium & $151 / 394 / 545$ & $65.65 / 62.9$ \\
\hline & Top & $16 / 187 / 203$ & $6.96 / 23.4$ \\
\hline & Total & $230 / 866$ & $100 / 100$ \\
\hline
\end{tabular}

conducted. Data collectors were also asked permission from respondents' parent and their children's to gain full commitment about the study. Parents or caregiver were given clear information about the study and each voluntary participant was sign out on consent prepared in local language. The informed consent of all study participants was obtained prior to the commencement of the study. All data was treated in a way that protects the confidentiality and of the participants involved in the study.

\section{Results and Discussion}

\section{Results}

Analysis (Table 1, Figure 1 and Figure 2).

\section{Discussion}

Enuresis is known as worldwide children developmental problem characterized by repeated voiding of urine into bed or cloths whether involuntary or intentional, the behavior is clinically significant as manifested by either a frequency of twice a week for at least 3 consecutive months for children age greater than 5 years.

In this study that considered the DSM-V as the criterion of enuresis in Adama city a total of 866 children with their families are interviewed. The assessment shows that the prevalence rate of enuresis in Adama city among children age $6-15$ was $26.6 \%$. Worldwide its prevalence extends to 1.4 to $28 \%$ in children age between 6-12 years varying from country to country. The studies conducted in Italy, Kenya, Saudi Arabia, China, and urban Ethiopian reported the prevalence of enuresis as $3.8,14.5,15,13,16.2$ percent respectively. These differences could be attributed to the differences in sample size, sampling method, age range, weather type, economic difference, tolerance for symptom in certain culture and definition of enuresis based on the DSM-IV or the ICD 10 criteria.

Age is a significant factor in enuresis in which its prevalence decreases with increasing age. Similarly in this study prevalence of enuresis among 7 years children is $30 \%, 10$ years children is $25 \%$ and $20 \%$ among 14 years children. The study stated in Kaplan book reported that by age 7 years prevalence of enuresis is $15.2 \%$, by age 10 , the overall prevalence of enuresis was reported to be 3 percent. The rate drops drastically for teenagers of 14 years to be a prevalence of 1.5 percent. The mean age for this study among children age is 9.95.

Prevalence of enuresis is more common among males than female. According to this conducted study in Adama city enuresis prevalence among age 5-15 years is $76.09 \%$ [175] for boys and 23.91 [55] among girl children. The study conducted in 2009 in the same city reveals the prevalence rate of enuresis $56 \%$ of them were boys and $44 \%$ of them were girls. In Iran, the 


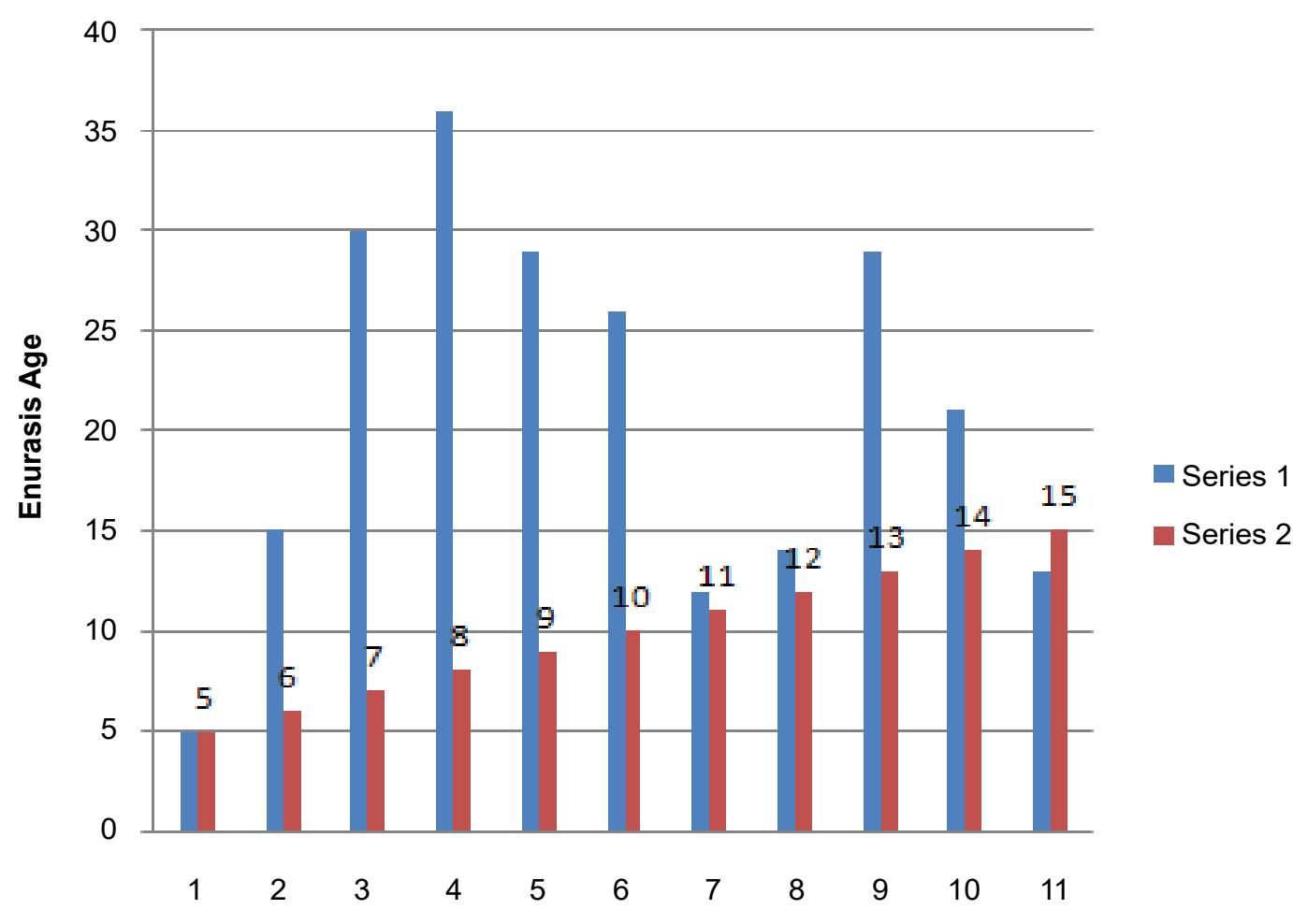

Axis Title

Figure 1: Histograph showing prevalence of enuresis among age group.

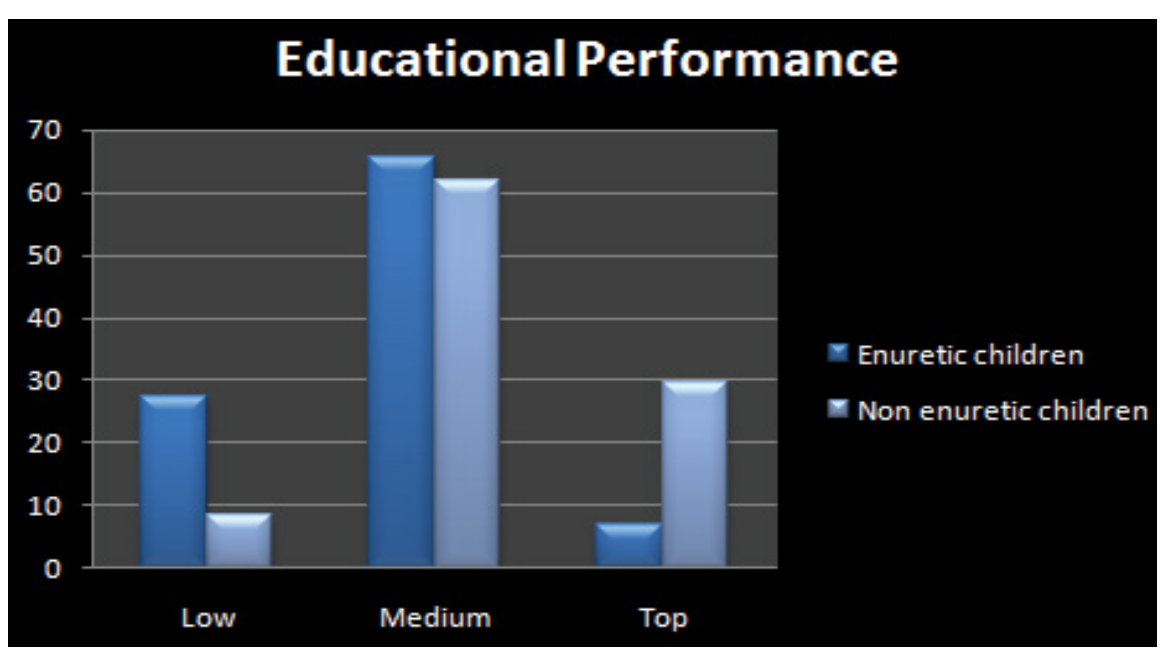

Figure 2: Histograph showing educational performance of enuretic and non-enuretic children.

prevalence of enuresis is $17.18 \%$ for boys and $11.82 \%$ for girls. In Ankara, Turkish study the general enuresis prevalence was $9.9 \%$ for males, as $9.2 \%$ for females however the sample size is different in all cases. In Egypt the prevalence of enuresis was not significantly higher in males $51.9 \%$ than females $48.1 \%$.

A positive family history of enuresis was seen in $28.57 \%$ children in India [21]; Community based study in Adama city in 2009 shows the positive family history of $34 \%$, and in this study 23.7 of enuretic children do have positive family history of enuresis. When family histories were explored in cases of children with enuresis, it was found that the mothers of 55.13\% [113] had the problem of enuresis while $16.59 \%$ [34] father, $17.07 \%$ [35] siblings, $10.24 \%$ [21] cousin and $0.97 \%$ [2] had enuretic relatives. A similar study in Istanbul conveys $76.2 \%$ mother and $14.9 \%$ enuretic father. Thus, statistically, the presence of enuresis in the family history was seen to have had a markedly significant impact on the occurrence of enuresis in the child [24].

The finding from the study reveals educational level of enuretic children at kindergarten weigh $10.87 \%$ [25], 1-6 grade magnify $63.91 \%$ [147], $7-8$ grade $19.13 \%$ [44] and above $9^{\text {th }}$ grade children are enuretic to the point of $6.09 \%$ [14]. So that the prevalence is higher among 1-6 grade students where detachment from family 
and care givers are higher and minimal when the level adjustment is stable.

The result of analysis regarding educational performance of enuretic and non enuretic children reveals enuretic children academic performance is 23.39\%, 65.65, and 6.96\% for low, medium and top performance respectively. Non enuretic children perform $13.6 \%$ in low rate, $62.9 \%$ medium, and $23.4 \%$ of children performs top rank academically. In academic low performance enuretic children predominate; where as in top performance non-enuretic children perform high level academically, albeit both of them perform equally at medium level educational performance.

With regard to current living conditions of all enuretic children $95.22 \%$ [219] of them live with their parents, $2.17 \%$ [5] of them with their relatives, $0.44 \%$ [1] adoption and 2.17 lives with others. Marital status of those enuretic children state that 94.35\% [217] of them from married families, and $5.66 \%$ [13] of them their families are divorced, and others. Family constriction is one of the main reasons for enuresis among children. In this study $96.52 \%$ [222] enuretic children do have more than one sibling. The $69.13 \%$ [159] enuretic children's are second and above born in the family. The main reason may be stresses associate with jealousy anxiety that exist in the family where attentions of the families are diverted toward new born baby.

A Study was conducted on Prevalence and risk factors of enuresis in rural area of Asysiut University, in Egypt confirm by asking the caregiver of children how to deal with the problem of enuresis, $15.7 \%$ mentioned that they consult others and about $29.4 \%$ consult a physician, $30.4 \%$ punish their children, while $24.4 \%$ do nothing [24]. In this study as result of enuretic behavior $53.04 \%$ [122] of them receive modern treatment from physician, $1.35 \%$ [3] from traditional therapist and $3.04 \%$ [7] of them receive therapy from spiritual person. Family responses to enuretic children's are 83.31\% [193] angriness over their children, 5.22\% [12] suffer social isolation from others, and all enuretic children receive advice from their respective others.

Enuresis can be divided into primary enuresis and secondary enuresis. A child who was a continent for at least 6 months before the onset of the bedwetting is considered to have secondary enuresis [1]. When primary enuresis and secondary enuresis are reported, a secondary onset accounts for about $25 \%$ of cases. The prevalence of secondary enuresis as a percentage of all cases increases with age. In a cohort of New Zealand children, $7.9 \%$ developed secondary enuresis by the age of 10 years [3]. In this study primary enuresis encounter 91.3\% [210] and 8.7\% [20] for secondary enuresis from the total of 230 enuretic children in Adama city. The pathogenesis of primary enuresis is similar to that of secondary enuresis. In primary enuresis, psychological problems are almost always the result of the condition in only rarely the cause. In secondary enuresis, however, psychological problems are a possible cause, albeit not a common one [1,10-15].

\section{Conclusion}

The prevalence of enuresis in children's of 5-16 yearsold age in Adama city was higher than similar conducted study founded in the literature. Its prevalence is also higher among boys than girls' children. Moreover, positive family history, number of siblings in household, birth order in the family, minimal age, punishment of children by their parents, underlying medical and psychiatric illness, were identified as the associated risk factors for enuresis. Concomitantly the study reveals educational performance of enuretic children is lower than non-enuretic children. Therefore, taking preventive and therapeutic measures in training parents dealing with children are essential to control enuresis. This paper by no means should be substitute for professional medical care.

\section{Recommendations}

Childhood enuresis was common in the community but parents rarely sought medical attention. Though this study report on the prevalence of enuresis in 6-16 years-old children in Adama city stresses on the need for parental education and awareness about this problem.

It has great importance that health extension worker at woreda and keble level working at the primary care centers should establish efficient relationship with children and their family toward combating enuresis.

Since enuresis has a variable etiologic spectrum from a simple developmental problem to a serious urinary obstruction medical department and psychiatric clinic work hand in hand and should take detailed history, make comprehensive physical examination and manage the problem with underlying associated risk causes.

Town health bureau must prepare awareness creation day for health extension workers, authorized keble leaders and to families of enuretic children.

\section{Acknowledgment}

Researcher's gratitude and appreciation would goes to Adma Hospital Medical College institutional review board for their valuable contribution on this final research work.

\section{References}

1. Van Tijen N, Messer A, Namdar Z (1998) Perceived stress of nocturnal enuresis in childhood. British Journal of Urology 81: 98-99.

2. Amanda BK (2008) Helping children with nocturnal enuresis. AJN American Journal of Nursing 106. 
3. Sadocks K (2007) Concise textbook of clinical psychiatry. ( $3^{\text {rd }}$ edn), New York.

4. Robson M, Cendron M (2016) Enuresis practice, essentials, background, pathophysiology, International children condition society, USA

5. American Psychiatric Association (2013) Diagnostic and Statistical Manual of Mental Disorders, Fifth Edition. Arlington, VA, American Psychiatric Association.

6. Giel R, Van Luijk JN (1969) Psychiatric morbidity in a small Ethiopian town. Br J Psychiatry 115: 149-162.

7. Ashenafi Y, Kebede D, Desta M, Alem A (2001) Prevalence of mental and behavioural disorders in Ethiopian children. East Afr Med J 78: 308-311.

8. Fekadu D, Alem A, Hagglof B (2006) The prevalence of mental health problems in Ethiopian child laborers. J Child Psychol Psychiatry 47: 954-959.
9. Golberg $J(2016)$ Enuresis in children, Web MD medical reference, LLC.

10. Ismail A (2016) Bed wetting accidents, when parent kills, South Africa.

11. Warzak WJ (1993) Psychosocial Implications of Nocturnal Enuresis. Clin Pediatr 38-40.

12. Schulpen TWJ (1997) The burden of nocturnal enuresis. Acta Paediatrica 86: 981-984.

13. Kalyanakarishan $R$ (2008) Evaluation and treatment of enuresis. Am Fam Physician 78: 489-496.

14. Solanki AN, Desai SG (2013) Prevalence and risk factors of nocturnalenuresis among school age children in rural areas, AMC MET Medical College, Ahmedabad, and Gujarat, India.

15. Sadocks K (2007) Synopsis of psychiatry, Behavioral science/clinical psychiatry. (10 $10^{\text {th }}$ edn), New York. 